\title{
Prior metformin therapy and 30-day mortality in patients with acute respiratory distress syndrome: a nationwide cohort study
}

\author{
Tak Kyu Oh, In-Ae Song \\ Department of Anesthesiology and Pain Medicine, Seoul National University Bundang Hospital, Seongnam, Korea \\ Contributions: (I) Conception and design: All authors; (II) Administrative support: IA Song; (III) Provision of study materials or patients: TK Oh; (IV) \\ Collection and assembly of data: All authors; (V) Data analysis and interpretation: TK Oh; (VI) Manuscript writing: All authors; (VII) Final approval \\ of manuscript: All authors. \\ Correspondence to: In-Ae Song, MD, PhD. Department of Anesthesiology and Pain Medicine, Seoul National University Bundang Hospital, 166 \\ Gumi-ro, Bundang-gu, Seongnam 463-707, Korea. Email: songoficu@outlook.kr.
}

\begin{abstract}
Background: The pleiotropic effect of metformin prevents lung injury in animal models. However, metformin has a controversial effect on survival outcomes in patients with acute respiratory distress syndrome (ARDS). This study aimed to investigate the effect of metformin therapy before ARDS diagnosis on mortality in ARDS patients with diabetes mellitus (DM).

Methods: Medical records from the national database, stored and provided by the National Health Insurance Service (NHIS) in South Korea were used. All adult diabetic patients admitted to a hospital for ARDS treatment from January 1, 2013 to December 31, 2017 were included in this study. Metformin users were defined as those prescribed continuous oral metformin for $\geq 30$ days before ARDS diagnosis. All other patients were included in the control group.

Results: Of the 6,500 patients selected for the study; 2,876 patients were prior metformin users. After propensity score matching (PSM), a total of 5,752 patients (2,876 patients in each group) were included in the analysis. The hazard of 30-day mortality in metformin users was not significantly different compared to the control group [hazard ratio (HR), 1.05; 95\% confidence interval (CI), 0.97-1.14; $\mathrm{P}=0.154$ ]. The survival time by the log-rank test was not significantly different between metformin users and controls (median time, 39.0 vs. 42.0 days, respectively; $\mathrm{P}=0.735$ ).

Conclusions: This population-based cohort study showed no significant association between prior metformin therapy and 30-day mortality in ARDS patients with DM. Moreover, prior metformin therapy was not associated with increase in overall survival times in ARDS patients with DM.
\end{abstract}

Keywords: Critical care; diabetes mellitus (DM); metformin; respiratory distress syndrome, adult; intensive care units (ICUs)

Submitted Dec 30, 2019. Accepted for publication Mar 04, 2020.

doi: $10.21037 /$ apm.2020.04.25

View this article at: http://dx.doi.org/10.21037/apm.2020.04.25

\section{Introduction}

Acute respiratory distress syndrome (ARDS) is a clinical syndrome characterized by acute onset of hypoxemia, bilateral pulmonary infiltrations and respiratory failure (1-3). In patients admitted to the intensive care unit (ICU), the incidence of ARDS is $10.4 \%$ (4), and its mortality ranges from $11 \%$ to $87 \%$ (5). The global burden of ARDS is an important global health issue and appears to be high in high- and upper-middle-income countries $(6,7)$.

Metformin is a biguanide drug that is most commonly prescribed for the management of type 2 diabetes mellitus (DM) (8), due to its well-known hypoglycemic effect. Its 
pleiotropic effects might ameliorate inflammatory injuries in vivo based on its inhibition of the expression of proinflammatory factors in vitro (9-12). In several experimental animal models of acute lung injury, pretreatment with metformin preserves the alveolar-capillary permeability and decreases the occurrence and severity of acute lung injury in high-pressure ventilation (13). In a recent study, metformin reduced lipopolysaccharide-induced lung injury in a rat model and increased the expression of inflammatory factors. These mechanisms might be related to alleviating ARDS (14). However, there is not enough information on prior metformin therapy and mortality in patients with ARDS. A retrospective single-center cohort study reported no significant association between prior metformin therapy and 30-day mortality in ARDS patients with DM (15). However, the study was performed in 2005 with a small sample $(n=128)$ of ARDS patients with DM.

Therefore, this nationwide study of a large population aimed to investigate the association between metformin therapy before ARDS diagnosis and mortality in ARDS patients with DM. We hypothesized that prior metformin therapy would be associated with better survival outcomes in ARDS patients with DM compared to controls.

\section{Methods}

\section{Ethics statement}

This population-based cohort study was performed per the ethical standards of the institutional and national research committee at the Seoul National University Bundang Hospital (X-1903-531-902) and the Health Insurance Review and Assessment Service (NHIS-2019-1-274).

\section{Data source}

The medical records from the national database, which are stored and provided by the National Health Insurance Service (NHIS) in South Korea, were used. The NHIS is the country's key institution for protecting the health of the nation, and is responsible for the management of national health insurance which safeguards the nation from disease, provides long-term care, and enables comfortable aging. In South Korea, all disease diagnoses, drug prescription information, and procedures should be registered in the NHIS database. The extraction of data was performed by a medical record technician in the NHIS center, who had no conflict of interest associated with this study.

\section{Study population}

All adult ( $\geq 18$ years old) diabetic patients who were admitted to a hospital for ARDS treatment [International Classification of Diseases; ICD-10 codes of J80* and E10E14 (DM)] from January 1, 2013 to December 31, 2017 were included in this study. For patients with multiple admissions ( $\geq 2$ ) for ARDS treatment, only the most recent admission was considered for analysis. Patients with incomplete or missing data were excluded.

The ARDS patients with DM were divided into metformin users, defined as those who had been prescribed continuous oral metformin for $\geq 30$ days before ARDS diagnosis, and the control, group which included all other patients.

\section{Study endpoint}

The primary endpoint was any 30-day mortality after the initiation of ARDS treatment. The date of death of all ARDS patients was extracted until May 30, 2019, from the NHIS database. The secondary endpoint was overall survival time. The survival time was calculated from the date of initial ARDS treatment to date of death or May 30, 2019 for ARDS survivors.

\section{Potential confounder}

The potential confounders of the study results included demographic information (age and sex), place of residence (Seoul, metropolitan cities, and other), and income level in quartile ratio at ARDS diagnosis. The comorbidities by ICD-10 codes [hypertension (I10-I16), coronary artery disease $\left(\mathrm{I} 20^{*}-\mathrm{I} 25^{*}\right)$, cerebrovascular disease $\left(\mathrm{I} 60^{*}-\mathrm{I} 69^{*}\right)$, lung cancer (C30-C39), chronic kidney disease (N18*), dyslipidemia (E78.0), anemia (D64*), chronic obstructive pulmonary disease (COPD) $\left(\mathrm{J} 44^{*}\right)$, asthma $\left(\mathrm{J} 45^{*}\right)$, arrhythmia (I49*), and liver cirrhosis $\left(\mathrm{K} 74^{*}\right)$ ] present before ARDS diagnosis were also included as confounders in this study.

\section{Statistical analysis}

The baseline characteristics of the patients were presented 
as mean with standard deviation for continuous variables, and numbers with percentages for categorical variables. First, we performed propensity score matching (PSM), which is known to reduce confounders in observational studies (16). PSM was performed without replacement at 1:1 ratio with caliper 0.2 via nearest neighbor method. All covariates were included in the PSM, and logistic regression analysis was performed to calculate propensity scores (PSs) as a logistic model. The absolute value of standardized mean difference (ASD) was used to determine the balance before and after PSM, and all ASDs $<0.1$ were used to determine that all covariates were sufficiently balanced through PSM.

After PSM, Cox regression analysis was performed in PS-matched cohort to investigate the hazard function of prior metformin therapy in 30-day mortality after initiation of ARDS treatment in patients, as compared to the control group. The results of the Cox regression analysis were presented as hazard ratio (HR) with $95 \%$ confidence intervals (CIs). Next, we performed multivariable Cox regression analysis for 30-day mortality in the entire cohort of all ARDS and diabetic patients as a sensitivity analysis. From this sensitivity analysis, we investigated the results derived from PS-matched cohort to generalize to all diabetic ARDS patients in South Korea. The proportional hazards assumption of the Cox proportional hazards model was tested using the log-minus-log plots. C-statistics were used to identify the C-index of the multivariable Cox regression models. There was no collinearity between the variables in the model as the variance inflation factor was $<2.0$. Finally, the survival time before and after PSM was estimated using Kaplan-Meier estimator for up to 365 days, considering that all diabetic ARDS patients were followed up for at least 450 days in this study. The survival time in the two groups was presented as median time with $95 \% \mathrm{CI}$. The log-rank test was used to test statistical significance of difference in survival time between the two groups. All statistical analyses were performed using the $\mathrm{R}$ software (version 3.6.1 with $\mathrm{R}$ packages), and $\mathrm{P}<0.05$ was considered statistically significant.

\section{Results}

From January 1, 2013 to December 31, 2017; 14,921 adult patients were admitted 17,022 times to the ICU for treatment of ARDS. Among the admissions, 2,101 were excluded due to multiple $(\geq 2)$ admissions for ARDS treatment in a single patient. Only the last episode of hospital admission for ARDS treatment was included in the analysis. DM was diagnosed in 6,500 patients, and these patients divided into two groups; prior metformin users $(n=2,876)$ and controls $(n=3,624)$. After PSM, a total of 5,752 patients (2,876 patients in each group) were included in analysis (Figure 1). The results of comparison of characteristics between metformin users and control group before and after PSM were presented in Table 1. The ASDs of all covariates were below 0.1 , indicating that the two groups were sufficiently balanced after PS matching. Figure S1 shows the distribution of PSs before (A) and after (B) PSM. The distribution of PS between the two groups became similar after PSM.

\section{Survival analysis after PS adjustment}

Table 2 shows the results of survival analysis in the PSmatched cohort. The 30-day mortality in metformin users was $45.9 \%(1,320 / 2,876)$, and in the control group was $44.4 \%(1,278 / 2,876)$. In the Cox regression model of the PS-matched cohort, the hazard of 30-day mortality in metformin users was not significantly different compared to the control group (HR, 1.05; 95\% CI, 0.97-1.14; $\mathrm{P}=0.154)$. Figure 2 showed the Kaplan-Meir curve between metformin users and the control group before (A) and after (B) PSM. After PSM, the survival time was not significantly different $(\mathrm{P}=0.735)$ between metformin users (median time, 39.0 days; 95\% CI, 35.0-45.0) and the control group (median time, 42.0 days; 95\% CI, 38.0-49.0) by log-rank test.

\section{Sensitivity analysis in entire cohort}

Table 3 shows the results of uni- and multivariable Cox regression models for 30-day mortality in the entire cohort. The hazard of 30-day mortality in metformin users was not significantly different compared to the control group (HR, $1.07 ; 95 \%$ CI, 0.98-1.15; $\mathrm{P}=0.072)$. The C-index of the multivariable model was 0.79 (95\% CI, 0.78-0.79).

\section{Discussion}

This population-based cohort study using nationwide data shows that prior metformin therapy is not associated with 30-day mortality in ARDS patients with DM. This association was similar in both the PS-matched cohort and multivariable adjustment of the entire cohort. Moreover, there was no significant difference in overall survival time after initiation of ARDS treatment for up to 450 days between prior metformin users and control group. 


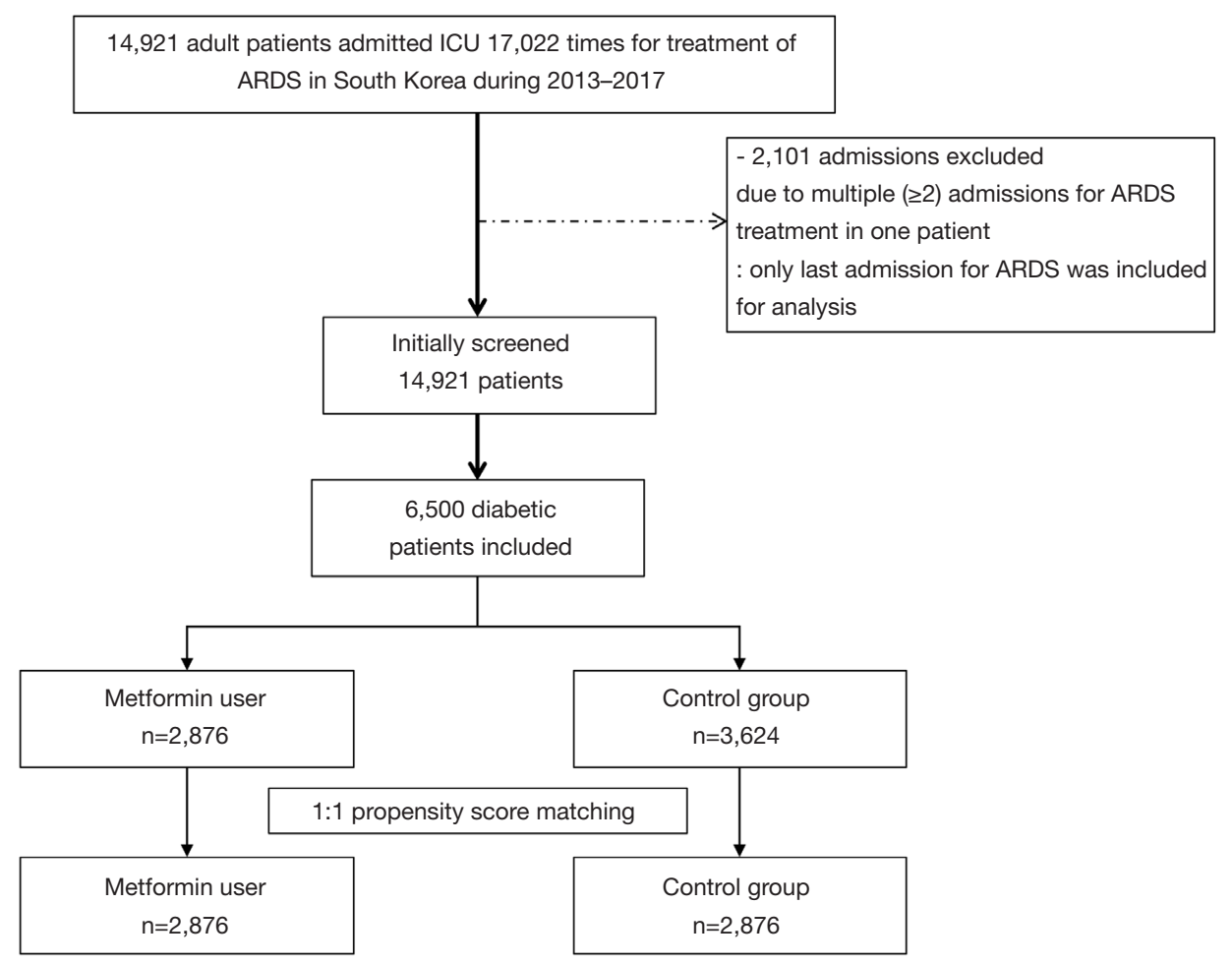

Figure 1 Patient selection flow chart. ICU, intensive care unit; ARDS, acute respiratory distress syndrome.

The results of this study coincide with the findings of a previous retrospective cohort study (15), suggesting that prior metformin therapy might not have potential benefits in ARDS patients with DM. However, previous animal studies report a reduction in lung injury by metformin therapy, possibly through various mechanisms $(13,17)$. The current study findings could be explained by the following speculations. First, even though patients with prior metformin therapy before ARDS diagnosis were selected, metformin was not administered after ARDS diagnosis because most of the patients were intubated for ventilator care. Thus, blood glucose levels might have been controlled by other drugs such as insulin, in most ARDS patients with DM. In ICU practice, insulin is commonly used to control blood glucose levels in critically ill patients with DM (18). Another study reported the beneficial effects of metformin in decreasing inflammatory cytokines when added to intensive insulin therapy (19). The result might be different if metformin therapy were to be continued after ARDS diagnosis in DM patients instead of insulin therapy.

Second, ARDS is one of the fatal clinical syndromes with a mortality of up to $90 \%$ after diagnosis (5). In our study, the 30-day mortality was approximately $45 \%$, and the median survival time in Kaplan-Meir curve was almost 40 days. Therefore, it is possible that the pleiotropic effect of prior metformin therapy might be limited in ARDS patients because there are other factors such as mechanical ventilation strategy (20) or prone positioning (21) that might be more closely related to prognosis in ARDS patients.

The differences between the current study and the previous retrospective cohort study must be carefully considered while interpreting the results (15). Both studies used PSM to adjust for confounders; however, the previous study included acute physiologic assessment, chronic health evaluation II scores, the simplified acute physiology score II scores, body mass index, heart rate, hemoglobin, lung injury score, and peak end-expiratory pressure. The current study did not include the above confounders due to limitation of data source. On the other hand, the current study included the income level, residence, and many other comorbidities before ARDS diagnosis, that were not included in the previous study. Despite the covariate differences in the PS model, it was notable that prior metformin therapy was not associated with 30-day mortality in both studies (15).

This study has several limitations. First, certain 
Table 1 Comparison of characteristics between metformin users and control group before and after PSM

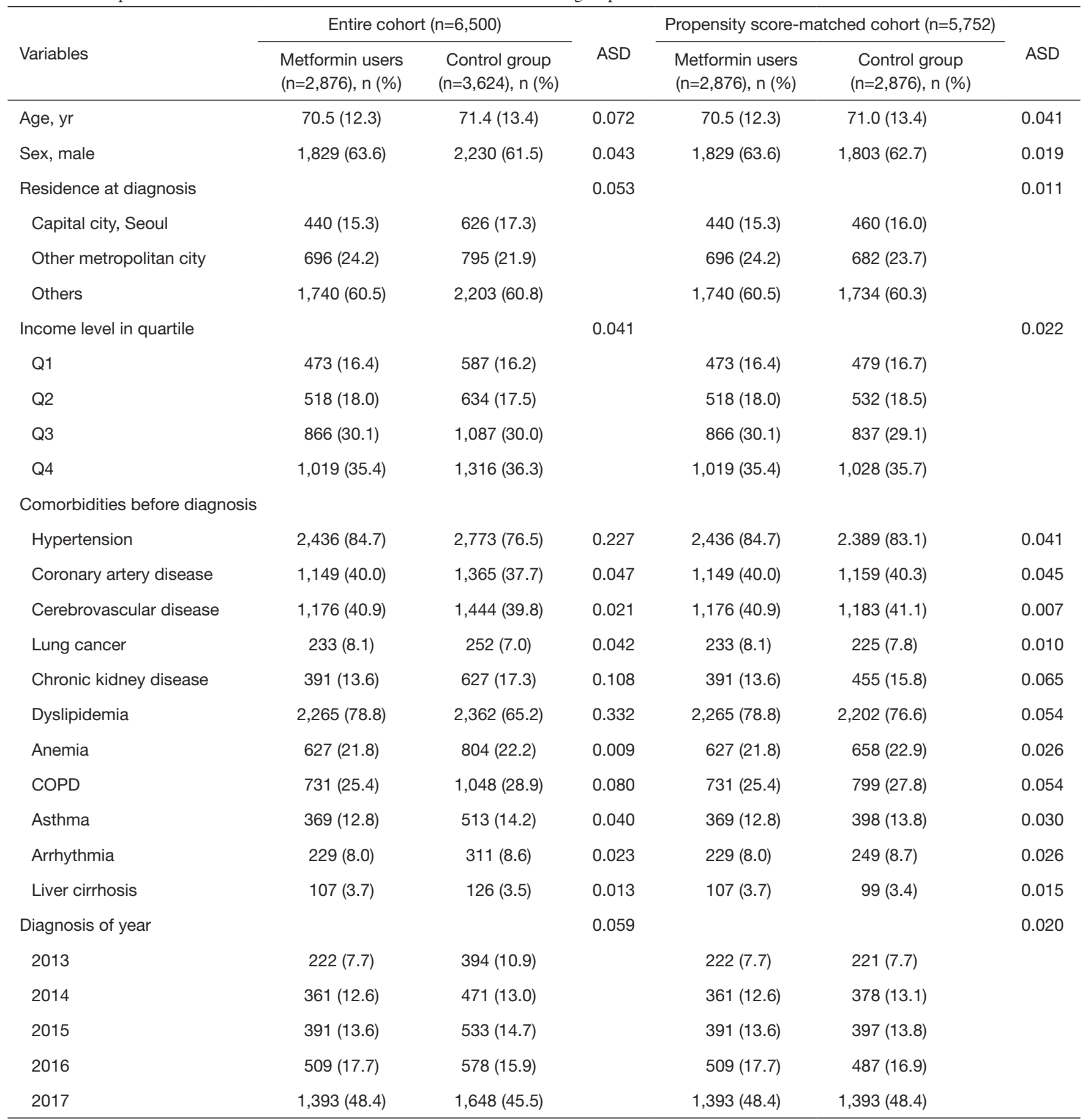

PSM, propensity score matching; ASD, absolute value of standardized mean difference; COPD, chronic obstructive pulmonary disease.

physiologic variables, such as body mass index, were not included in the analysis because the NHIS database does not contain this information. Second, we used the ICD-10 codes registered in the NHIS database to define comorbidities of ARDS patients; however there is a possibility that the diseases specified in the ICD-10 codes might have differed from the actual comorbidities of all ARDS patients with DM. Third, certain factors (the P/ 
Table 2 Survival analysis in propensity score matched cohort

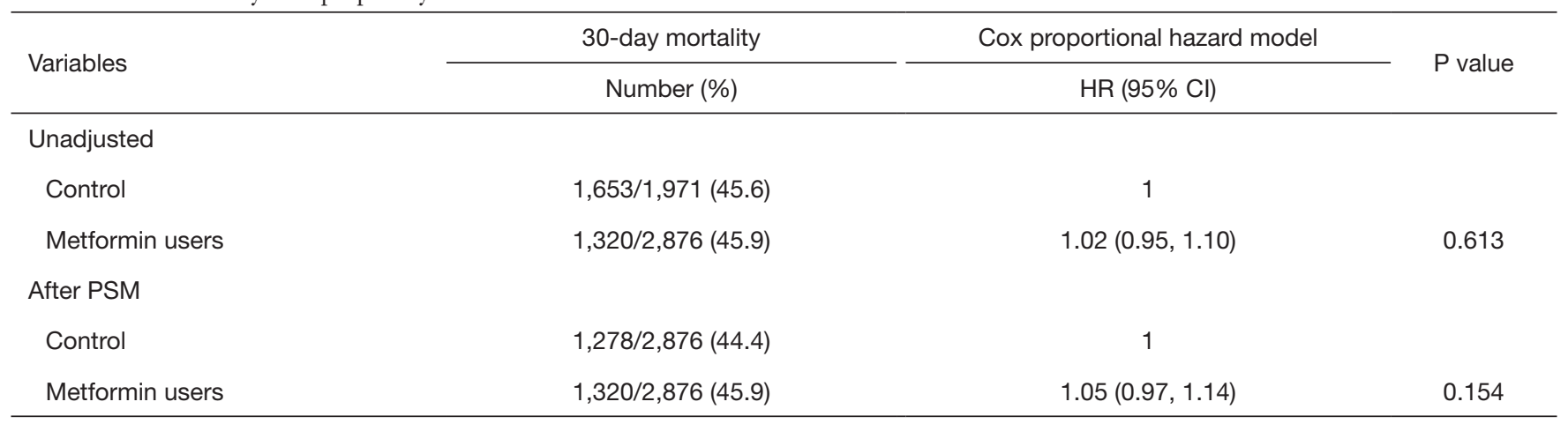

$\mathrm{HR}$, hazard ratio; $\mathrm{Cl}$, confidence interval; PSM, propensity score matching.

\section{A}

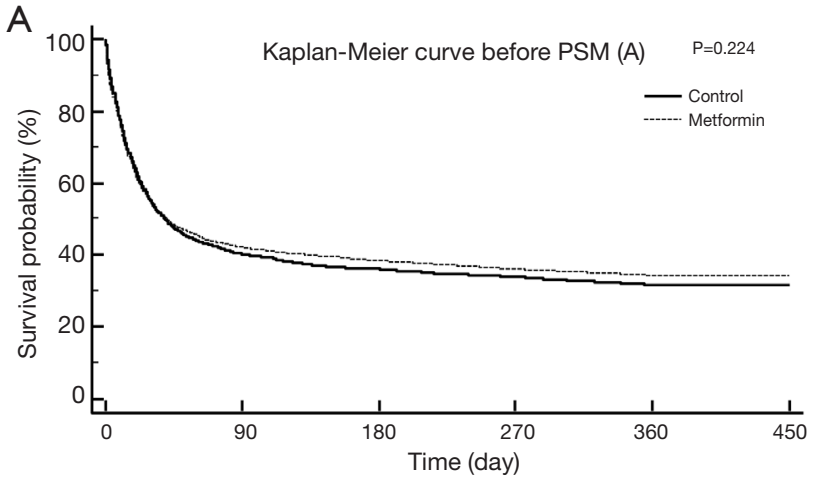

$\begin{array}{lllllll}\text { Control } & 3,624 & 1,457 & 1,306 & 1,225 & 1,145 & 1,091 \\ \text { Metformin } & 2,876 & 1,205 & 1,098 & 1,029 & 978 & 946\end{array}$

\section{B}
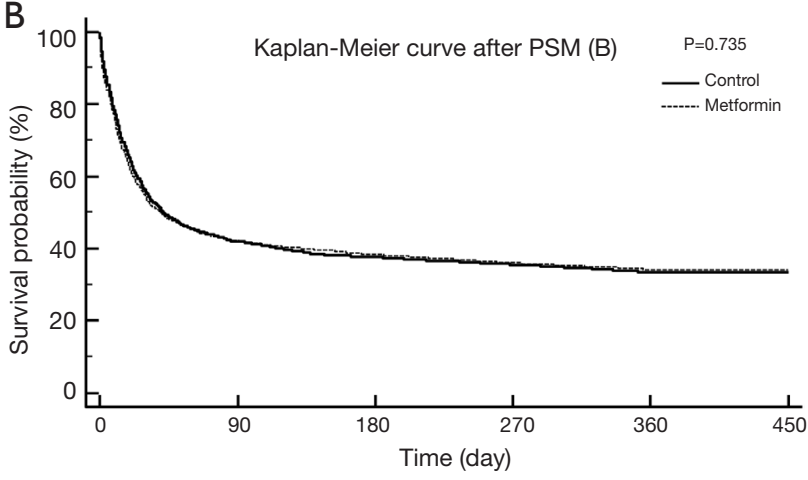

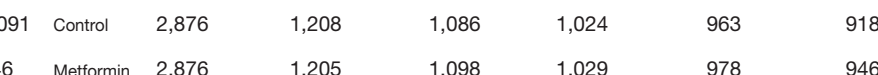

Figure 2 Kaplan-Meier curve of overall survival of metformin users and control group before (A) and after (B) PSM. PSM, propensity score matching.

F ratio, simplified acute physiology score-II score, the acute physiology and chronic health evaluation II score, and ventilator strategy) that are closely related to ARDS prognosis were not studied. The NHIS database contains data regarding prescription of drugs and procedures only. Lastly, PS adjustment and multivariable adjustment controlled only the known confounders. The possibility of unmeasured and unknown confounders cannot be excluded.

In conclusion, there is no significant association between prior metformin therapy and 30-day mortality in ARDS patients with DM. Moreover, prior metformin therapy was not associated with overall survival for up to 450 days in ARDS patients with DM. Thus, prior metformin therapy might have no benefit for survival improvement in ARDS 
Table 3 Uni- and multivariable Cox regression analysis for 30-day mortality in entire cohort

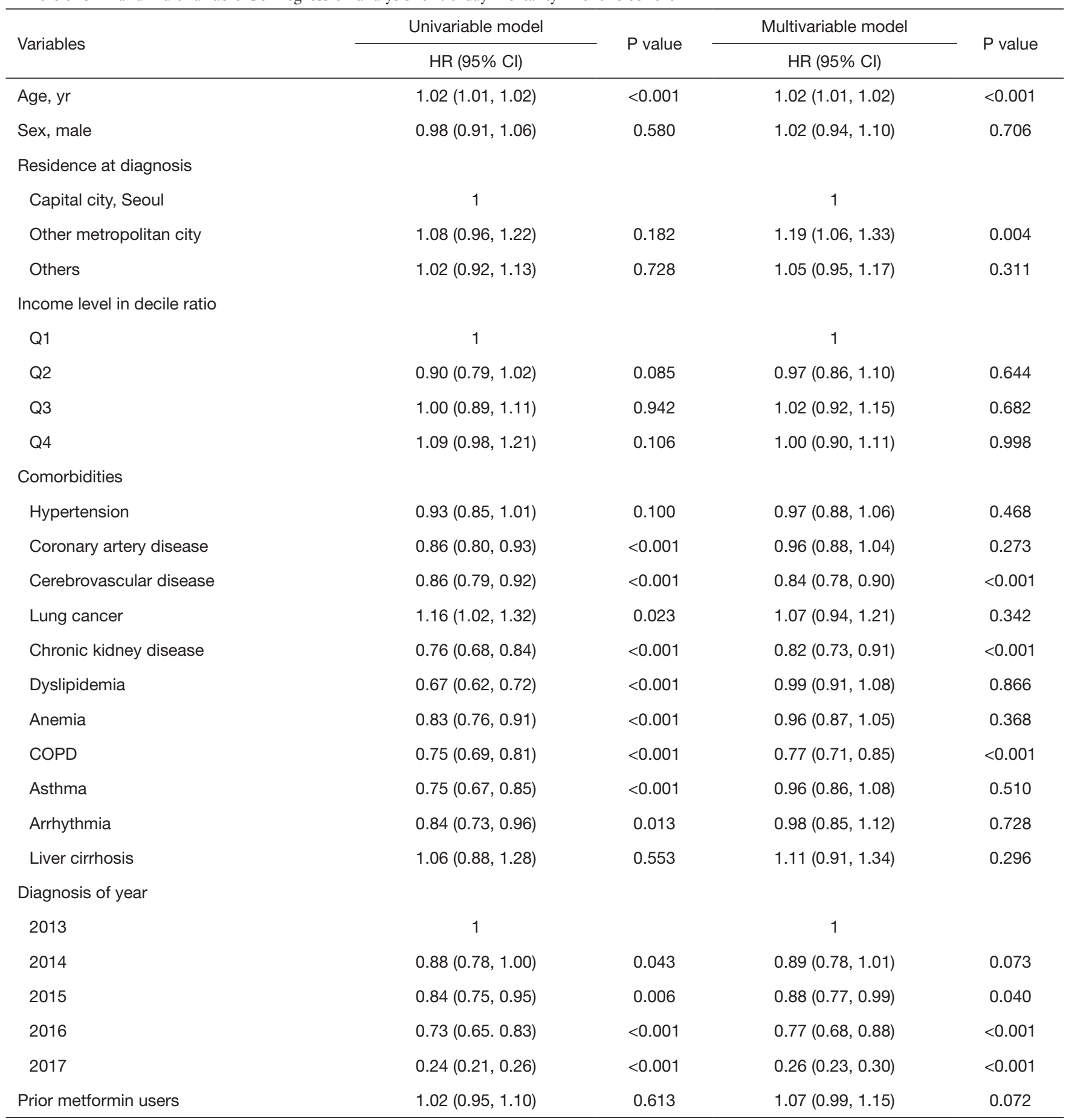

C-index: 0.79 (95\% Cl: 0.78, 0.79). HR, hazard ratio; Cl, confidence interval; COPD, chronic obstructive pulmonary disease. 
patients with DM. Future prospective studies are required to confirm these findings.

\section{Acknowledgments}

Funding: None.

\section{Footnote}

Conflicts of Interest: Both authors have completed the ICMJE uniform disclosure form (available at http://dx.doi. org/10.21037/apm.2020.04.25). The authors have no conflicts of interest to declare.

Ethical Statement: The authors are accountable for all aspects of the work in ensuring that questions related to the accuracy or integrity of any part of the work are appropriately investigated and resolved. This populationbased cohort study was performed per the ethical standards of the institutional and national research committee at the Seoul National University Bundang Hospital (X-1903531-902) and the Health Insurance Review and Assessment Service (NHIS-2019-1-274).

Open Access Statement: This is an Open Access article distributed in accordance with the Creative Commons Attribution-NonCommercial-NoDerivs 4.0 International License (CC BY-NC-ND 4.0), which permits the noncommercial replication and distribution of the article with the strict proviso that no changes or edits are made and the original work is properly cited (including links to both the formal publication through the relevant DOI and the license). See: https://creativecommons.org/licenses/by-nc-nd/4.0/.

\section{References}

1. Ashbaugh DG, Bigelow DB, Petty TL, et al. Acute respiratory distress in adults. Lancet 1967;2:319-23.

2. Bernard GR, Artigas A, Brigham KL, et al. The American-European Consensus Conference on ARDS. Definitions, mechanisms, relevant outcomes, and clinical trial coordination. Am J Respir Crit Care Med 1994;149:818-24.

3. ARDS Definition Task Force, Ranieri VM, Rubenfeld GD, et al. Acute respiratory distress syndrome: the Berlin Definition. JAMA 2012;307:2526-33.

4. Bellani G, Laffey JG, Pham T, et al. Epidemiology, patterns of care, and mortality for patients with acute respiratory distress syndrome in intensive care units in 50 countries. JAMA 2016;315:788-800.

5. Máca J, Jor O, Holub M, et al. Past and present ARDS mortality rates: a systematic review. Respir Care 2017;62:113-22.

6. Adhikari NK, Fowler RA, Bhagwanjee S, et al. Critical care and the global burden of critical illness in adults. Lancet 2010;376:1339-46.

7. Riviello ED, Kiviri W, Twagirumugabe T, et al. Hospital incidence and outcomes of the acute respiratory distress syndrome using the kigali modification of the berlin definition. Am J Respir Crit Care Med 2016;193:52-9.

8. Bailey CJ. Metformin: historical overview. Diabetologia 2017;60:1566-76.

9. Kim SA, Choi HC. Metformin inhibits inflammatory response via AMPK-PTEN pathway in vascular smooth muscle cells. Biochem Biophys Res Commun 2012;425:866-72.

10. Kuo CL, Ho FM, Chang MY, et al. Inhibition of lipopolysaccharide-induced inducible nitric oxide synthase and cyclooxygenase- 2 gene expression by 5 -aminoimidazole-4-carboxamide riboside is independent of AMP-activated protein kinase. J Cell Biochem 2008;103:931-40.

11. Liu G, Wu K, Zhang L, et al. Metformin attenuated endotoxin-induced acute myocarditis via activating AMPK. Int Immunopharmacol 2017;47:166-72.

12. Yuan H, Li L, Zheng W, et al. Antidiabetic drug metformin alleviates endotoxin-induced fulminant liver injury in mice. Int Immunopharmacol 2012;12:682-8.

13. Tsaknis G, Siempos II, Kopterides P, et al. Metformin attenuates ventilator-induced lung injury. Crit Care 2012;16:R134.

14. Yu LL, Zhu M, Huang Y, et al. Metformin relieves acute respiratory distress syndrome by reducing miR-138 expression. Eur Rev Med Pharmacol Sci 2018;22:5355-63.

15. Jo YS, Choi SM, Lee J, et al. Effect of preadmission metformin use on clinical outcome of acute respiratory distress syndrome among critically ill patients with diabetes. Tuberc Respir Dis (Seoul) 2017;80:296-303.

16. Rosenbaum PR, Rubin DB. The central role of the propensity score in observational studies for causal effects. Biometrika 1983;70:41-55.

17. Zhang X, Shang F, Hui L, et al. The alleviative effects of metformin for lipopolysaccharide-induced acute lung injury rat model and its underlying mechanism. Saudi Pharm J 2017;25:666-70.

18. Clain J, Ramar K, Surani SR. Glucose control in critical 
care. World J Diabetes 2015;6:1082-91.

19. Ansari G, Mojtahedzadeh M, Kajbaf F, et al. How does blood glucose control with metformin influence intensive insulin protocols? Evidence for involvement of oxidative stress and inflammatory cytokines. Adv Ther 2008;25:681-702.

20. Acute Respiratory Distress Syndrome Network, Brower

Cite this article as: Oh TK, Song IA. Prior metformin therapy and 30-day mortality in patients with acute respiratory distress syndrome: a nationwide cohort study. Ann Palliat Med 2020;9(3):903-911. doi: 10.21037/apm.2020.04.25
RG, Matthay MA, et al. Ventilation with lower tidal volumes as compared with traditional tidal volumes for acute lung injury and the acute respiratory distress syndrome. N Engl J Med 2000;342:1301-8.

21. Scholten EL, Beitler JR, Prisk GK, et al. Treatment of ARDS With Prone Positioning. Chest 2017;151:215-24. 


\section{Supplementary}

A

Distribution of PSs in metformin users before PSM (A)

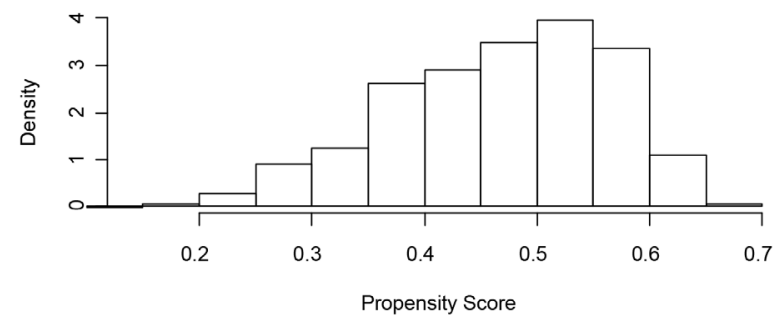

Distribution of PSs in control group before PSM (A)

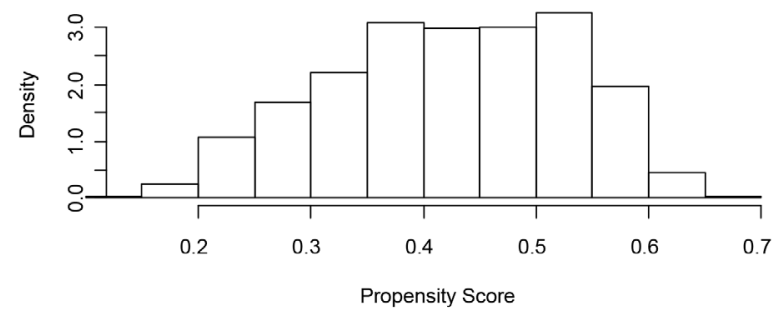

B Distribution of PSs in metformin users after PSM (B)

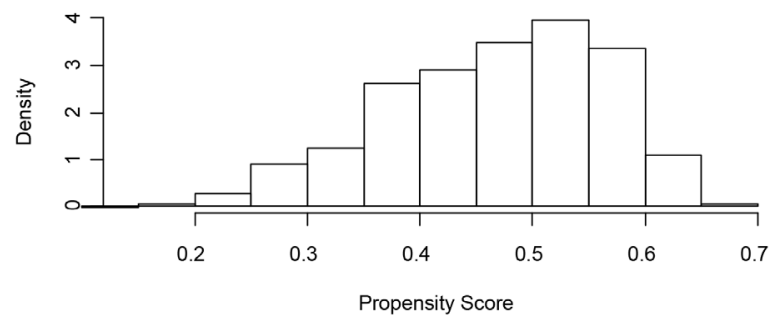

Distribution of PSs in control group after PSM (B)

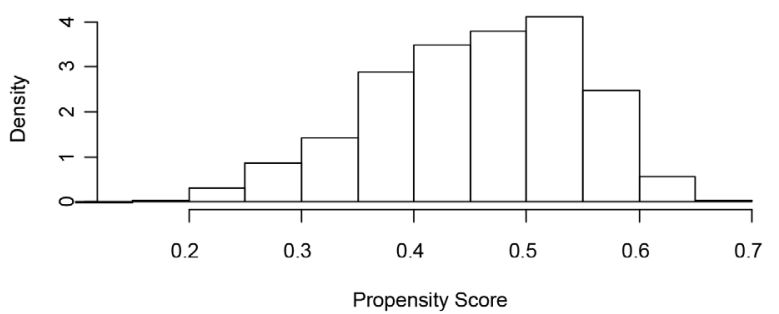

Figure S1 The distribution of PSs before (A) and after (B) PSM. PSs, propensity scores; PSM, propensity score matching. 